\title{
Simulation on Collective Panic Behavior Based on PI
}

\author{
Wen ZHENG, Da ZHU \\ (Management Department, Northeastern University at Qinhuangdao, Qinhuangdao, 066004, P. R. China \\ zhengwen@neuq.edu.cn, 1433137831@qq.com
}

\begin{abstract}
The collective panic behavior, which is caused by PI (Propagation Intensity), influences the welfare of citizens and the stability of the society (business). A well-known challenge is to understand the spreading mechanism of the panic behavior. In this work, the $\mathrm{PI}$ is classified as $f$ and $g$, defined in the coefficient $f$ (Promotion Intensity) and $g$ (Inhibition Intensity) in the probability. MAS (multi-agent-simulation) model/tool is used to construct the systematic structure composed of four types of the participants (the individuals, the mass media, the social leaders, and the supervisors).This tool stimulates the collective panic behavior from the initial coefficient $f$ and $g$, showing the different results in the condition of the population size and the conformity. Through setting up the rules and the strategies in an emergency, the framework stimulates the interaction between the agents (e.g. between the individuals, between the individuals and the mass media, between the individuals and the social leaders, between the individuals and the supervisors). The results advocate that the current simulator might be an appropriate first step towards the simulation and analysis of the combination of rules and strategies in panic behavior.
\end{abstract}

Keywords: Collective Panic Behavior, MAS(Multi-Agent-Simulation), PI(Propagation Intensity), NetLogo Platform

The project is funded by National Natural Science foundation, Code NO. 71473032.

\section{Introduction}

Nowadays, understanding the spreading mechanism of individual irrational behavior and the collective panic behaviors in an emergency is still an open challenge. China is a relation-society and the people in this country will share their feelings in an incredible way. When an emergency comes, a rumor is like a gem(seed). It can be spread heavily in a positive or negative way. It is absurd that many people rushed to buy the salt in China after the nuclear leakage accident in Fukushima (Japan)in case of the rumor" using iodized salt can prevent the harm of iodine -131" in 2011. The rumor (three days without daylight) leaded to the panic buying of the candles in some cities in China in 2012. What is the reason and the mechanism of the abnormal, large-scale panic behavior for some certain ordinary situations in an emergency? The current work presents an MAS tool, which aims at stimulating and analyzing the collective panic behavior in an emergency. The paper concludes four parts. First, we give the background of the study. Second, the framework of the CPBsim (Collective Panic Behavior Simulation) is given. Third, MAS model is constructed to explore the impact of the PI(Propagation Intensity). Last, it gives some discussions and conclusions. 
Wen ZHENG, Da ZHU; Simulation on Collective Panic Behavior Based on PI, Transactions on Networks and Communications, Volume 6 No. 6, December (2018); pp: 57-75

\section{Background}

\subsection{Literature Review}

In the literature, the researches on the abnormal phenomena of panic behavior had been analyzed before this study(Chen et al, 2007; Dai et al, 2015; Kou et al, 2012).They can be classified among: (a) the ones, those proposed the practical cases for the panic behavior,(Thomas et al, 2002;Edward et al, 2005; Li et al, 2009), and (b) the ones, those analyzed the different facets of the theoretical models in mathematics(Bergemann et al, 1996; Barabasi et al, 1999; Kirman et al, 2001;Yuan et al, 2005; Izquierdo et al, 2005), and (c) the studies based on the model of the simulation in individuals(Du et al, 2011; Zhang et al, 2011; Liu et al, 2014; Zhang et al, 2014;Zheng et al, 2016; Carlos et al, 2016). The practical cases are as following: after terrorist attacking in the "9.11", the purchase of gas masks in the shopping centers and the ciprofloxacin and other drugs in drug stores in United States increased rapidly(Thomas et al, 2002). The phenomenon of gasoline buying came after Hurricane Karina hit the United States(Edward et al, 2005). Some other theoretical researches in mathematics gave answers in, such as: establishing and solving the consumer decision-making behavior model to explain the phenomena of collective buying from the economic point of view(Yuan et al, 2005), and establishing the model and simulating the impact on the psychological expectations of panic behavior in context of an emergency (Sun et al,2006; Dai et al, 2015; Zhuang et al, 2013; Zhang et al, 2015; Kou et al, 2012; Liu et al, 2014; Chen et al, 2007; Qiang et al, 2014; Chang et al, 2011). The risk of the perceptions as the core of the intermediary variables would have a significant impact on people's mental state and the process of individual decision-making behaviors in the tsunami, the earthquake and other disasters (Li et al, 2009; Liu et al, 2013; Xu, 2003; Bi, 2007; Li, 2008). A three-level index evaluation system was established, pointing out the characteristics of the individual behaviors in an emergency and considering the external material environment and the individual's personality traits and other related factors(Zhao et al, 2010). The microscopic discrete dynamics concept model was deduced in the Internet surrounding in analyzing the interrelationship between Internet users' behaviors and the social dynamics factors. It can quantify the perceived information of the Internet users(He et al, 2010). Other researchers introduced the group behavior dynamics in constructing the dynamic theoretical model of the evolution of the collective behavior. (Wei et al, 2011; Xie et al, 2013; Lu et al, 2011). The modeling method was used in the microscopic perspective in constructing the simulation model of individual, revealing the behavior of individual and the interaction among them, as well as the whole situation of rumor propagation(Liu et al, 2014; Du et al, 2011; Zhang et al, 2016; Zhang et al, 2014; Zhang et al, 2011). Furthermore, the population model under the uncertain environment was done by proposing the fuzzy membership functions for the uncertainty movement of the microcosmic individuals(Chen et al, 2007).Based on the bounded trust rules, the conceptual model of noisy heterogeneous bounded trust was established(Zhao et al, 2015). With the method of Multi-Agent modeling, the public opinion evolution based on Internet were constructed(Du et al, 2015; Zhang et al, 2011; Yang et al, 2010; Chen et al, 2015).

The existing researches showed the collective panic behavior, which was caused by the rumors in context of an emergency. From the perspective of the systematic dynamics, the existing work explored the influence of the effective transmission intensity of rumors on the different amount of the participants in 
Transactions on Networks and Communications; Volume 6, No. 6, Dec 2018

the collective panic behavior. The paper is based on the NetLogo platform on giving $t$ the simulation results.

\subsection{Assumption}

1) In an emergency situation, individuals, who are in the initial population of the spatial range (Li et al,2014), are divided into two categories: the participants with the collective panic behavior or with noinvolving in it. The participants, who are in the collective behavior, are not only in joining in but also in affecting the surrounding neighbors. The participants at $t$ time accounts for the Initial Population Size(IPS) $s(t)$. The ones, who do not participate in the panic behavior at $t$ time, account for the proportion of the initial population in $1-s(t)$. The potential degree, which will be affected by the collective panic behavior of the population size, is basically stable. The initial population size is $W$.

2)The concentration of the population in the space is large and the spatial distribution is relatively uniform. The number of the neighbors of each individual is tiny, and at any time $t$, each participant of the panic behavior can communicate with the $k$ individuals in the initial population of the space.

3) The collective panic behavior is affected by $\mathrm{PI}$, which is cited as the positive one as the promotion intensity and the negative one as the inhibition intensity. And PI i expressed as the promotion intensity $f(r, e)$ and the inhibition intensity $g(c, l)$, respectively. Where $r$ is the credibility of the rumor, $e$ indicates the degree of the negative impact of the panic event itself, $c$ represents the credibility of the government administration, and 1 indicates the disposition of the government administration. $f(r, e)$ and $g(c, l)$ are monotonically increasing functions of $r, e$ and $c$. (In scope of $0 \leq r, e, c, l \leq 1$, $0 \leq f(r, e) \leq 1, \quad 0 \leq g(c, l) \leq 1)$.

4) Assuming that the probability of the initial group does not participate in the panic behavior(Yan et al, 2010; Zheng et al, 2016). The participants are only related to the credibility of the rumors and the negative impact of the panic (Virginia et al, 2016) event itself. The value is the proportion of $f(r, e)$. In the initial group, the probability of panic behavior participants become the withdrawal from the panic and the credibility of government administration, as well as the intensity of disposal of government departments, and its value is proportion of $g(c, l)$. 


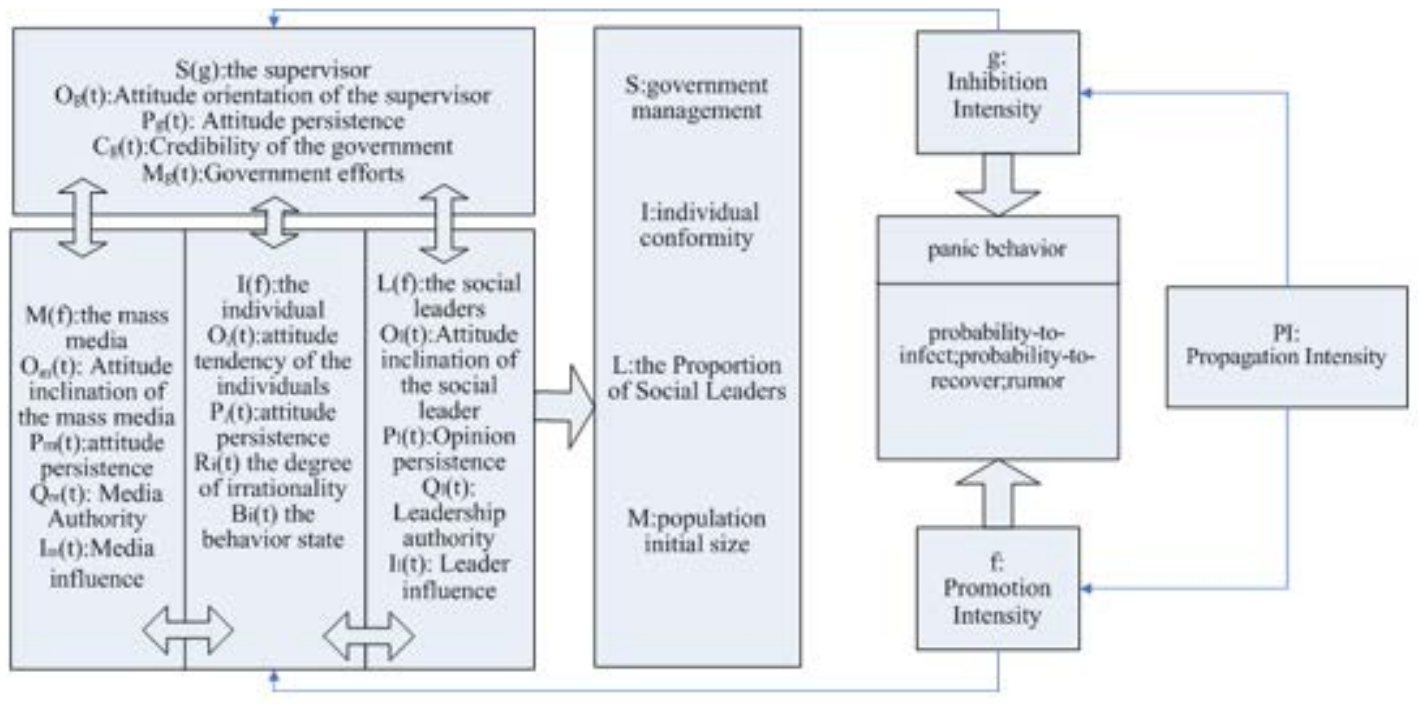

Fig.1 Class diagram of the CPB/Panbeh-agents

\section{CPBsim/PanbehSim}

CPBsim/PanbehSim is the simulation tool on the framework of the Collective Panic Behavior.

\subsection{The Agents}

\subsubsection{Individual}

The value is within continuous range.

1) Attitude tendency of the individual: $O_{i}(t)$ indicated the attitude of individual $i$ to panic behavior at moment $t$. Attitude tendency is divided into three kinds: negative, neutral, and positive. The value is in continuous range, and the initial attitude tends to uniform random distribution.

2) Attitude persistence: $P_{i}(t)$ indicates the persistence degree of the attitude of individual $i$ towards the panic at moment $t$. Attitude persistence is divided into three categories: lower, general, and higher. It is within the continuous range.

3) The degree of irrationality: $R_{i}(t)$ represents the degree of irrationality of the individual $i$ at time $t$. The value is in continuous range.

4) The behavior state: $B_{i}(t)$ represents the behavior state of the individual $i$ at time $t$. Behavior states are divided into three types: (1)it does not yet involve in the collective panic behavior;(2)it has involved in the collective panic behavior; (3)it has withdrawn from the collective panic behavior.

\subsubsection{Mass Media}

The value is in the continuous ranges. 
Transactions on Networks and Communications; Volume 6, No. 6, Dec 2018

1) Attitude inclination of the mass media: $O_{m}(t)$ represents the attitude of the mass media $\mathrm{j}$ at the $t$ moment to the information (the facts or the rumor), which is in contacted with. The value is in continuous intervals.

2) Attitude persistence: $P_{m}(t)$ stands for the degree to which the mass media $j$ maintains its attitude toward the information (facts or rumor). The value is in the continuous intervals.

3) Media Authority: $Q_{m}(t)$ represents the authority size of the mass media $j$ at the $t$ moment. The value is in the continuous range.

4) Media influence: $I_{m}(t)$ represents the spatial range, which the mass media $j$ can influence the group at the $t$ moment.

\subsubsection{Social Leader}

1) Attitude inclination of the social leader: $O_{l}(t)$ stands for social leaders' attitudes towards their own exposure to information (facts or rumors) at $t$. The values are in the successive intervals.

2) Opinion persistence: $P_{l}(t)$ refers to the degree to which social leaders adhere to their information (facts or rumors) set at 1.

3) Leadership of the authority: $Q_{l}(t)$ shows the authority size of the social leaders at the $t$ moment in the continuous range of the values.

4) Leader influence: $I_{l}(t)$ represents social leaders' influence at the $t$ moment in the groups in the continuous range of the values.

\subsubsection{Supervisor}

1) Attitude orientation of the supervisor: $O_{g}(t)$ indicates the attitude of the supervisor to the information that they have at the $t$ moment. The government is positive in the process of the collective panic behavior.

2) Attitude persistence: $P_{g}(t)$ stands for the degree to which government authorities adhere to the information they spread.

3) Credibility of the government: $C_{g}(t)$ means that the confidence of the individual in the crowd to the government management will change with the management of the panic incident, and take the value in the continuous range.

4) Supervisor's efforts: $M_{g}(t)$ stands for the government's management of the negative information (rumors and related rumors) to deal with the intensity, in the continuous range of $[0,1]$.

\subsection{The Rules}

\subsubsection{Rule 1}

$$
\text { agent } \in\{\Omega, B, C, E, \Psi, F\} \text {, }
$$

1) $\Omega\left(\Omega=\left\{\right.\right.$ agent $_{1}$, agent ${ }_{2}, \cdots$, agent $\left.\left._{W}\right\}\right)$ is the whole group space, which is composed of a collection of all agents.

2) $B\left(B=\left\{b_{0}(t), b_{1}(t)\right\}\right)$ is the individual behavior strategy state space. $\mathrm{b}_{0}(\mathrm{t})$ indicates that the individual takes the panic behavior without taking part in at the $t$ moment. And $\mathrm{b}_{1}(\mathrm{t})$ stands for 
the individual at the $t$ moment to take part in the panic behavior. Three agents, which are indicated in colors of green, red, and gray on programs. Green indicates that the behavior of the agent is not yet involved. Red indicates that the behavior of the agent is participating. Grey indicates that the agent has withdrawn from the panic behavior.

3) $C(C=\{c(1), c(2)\})$ represents the possibility that individuals are infected by the other panic neighbors. Among them, $c(1)$ is a rational type, and $c(2)$ is an irrational type.

4) $E(E=\{f, g\})$ is the external environment function, where $f(0 \leq f \leq 1)$ is the promotion intensity, and $g(0 \leq g \leq 1)$ is the inhibition intensity.

5) $\Psi\left(\Psi=\left\{\Psi_{1}, \Psi_{2}, \cdots, \Psi_{k}\right\}\right)$ is the space function of the agents and their neighbors.

6) $F\left(F:\left\{\left(C_{i}, E_{i}, \Psi_{i}\right) \rightarrow B_{i}(t)\right\} \times t \rightarrow B_{i}(t+1)\right)$ is the behavior state transition function. The behavior state of agent at $t=1$ is related to its own individual characteristics, surrounding neighbors, and external social environment at the $t$ moment.

7) $t(t=\{1,2,3, \cdots\})$ is the system time pointer. During the evolution of the simulation system, $\Omega$ and $\Psi$ are fixed, and the parameters of $B$ are changed with the simulation system clock. $C$ and $E$ are the external adjustable parameters of the simulation system. And they are given before the simulation system is running. Because of the individual learning, the behavior strategy of the agent will take in the collective panic behavior under the circumstances of an emergency. The system is designed as a learning mechanism in updating rules, which take consideration the status of the individual, the neighborhood, and the external social environment.

\subsubsection{Rule 2}

The characteristics of the decision-making of the indifferent rationality and the herd irrational individual are recorded as $c(i),(i=1,2), c(1), c(2) \sim U(a, b)$, and ordered as $c(1) \sim U(0,0.5), c(2) \sim U(0.5,1)$.

The size of $C$ reflects the size of individual conformity, which means the smaller value of $C$ is, the less likely it is to learn from the surrounding individuals. The greater value of $C$ is, the more it is likely to learn from the surrounding individuals.

\subsubsection{Rule 3}

The trait of individual conformity is $q_{i}$, and $n$ is the total number of neighbors of the individual ${ }^{a g e n t} t_{i}$. $\sum^{n} b_{i k}{ }^{(0)}$ indicates the number of neighbors who do not participate in the panic behavior.

Individual changes from the panic behavior to withdrawal from the panic behavior. The renewal probability is:

$$
P\left\{b_{1}(t) \rightarrow b_{0}(t+1)\right\}=\left\{\begin{array}{cc}
P_{1} & P_{1}>0 \\
0 & P_{1} \leq 0
\end{array}\right.
$$

The update probability of the behavioral strategy of the individual who changes from the no-involving one in the panic behavior to the involving one is: 


$$
\begin{aligned}
& P\left\{b_{0}(t) \rightarrow b_{1}(t+1)\right\}=\left\{\begin{array}{cc}
P_{2} & P_{2}>0 \\
0 & P_{2} \leq 0
\end{array}\right. \\
& P_{1}=q_{i}\left[\frac{1}{n} \sum_{k=1}^{n} b_{i k}{ }^{(0)}+(g-f)\right] \\
& P_{2}=q_{i}\left[\frac{1}{n}\left(n-\sum_{k=1}^{n} b_{i k}{ }^{(0)}\right)+(f-g)\right]
\end{aligned}
$$

In (3) and (4), $q_{i}$ stands for the subjective character of the individual to reflect the possibility of the individual conformity, which is affected by the panic infection.; $\frac{1}{n} \sum_{k=1}^{n} b_{i k}{ }^{(0)}$ represents the proportion of neighbors in the neighborhood of agent $_{i}$, who do not participate in the panic behavior strategy. $\frac{1}{n} \sum_{k=1}^{n} b_{i k}{ }^{(0)}$ reflects the local spatial environment of the individual; $g-f$ is the difference between the inhibition intensity and the promotion intensity, and reflects the external social environment.

Simulation system in the initial state, according to the above rules to update the evolution, if most of the agent's behavior strategy tends to participate in panic behavior strategy, the collective panic behavior is formed.

\subsection{The Interactions among the Agents}

In simulation model of the Multi-Agent of collective panic behavior, individual behavior changes are influenced by attributes of their own, of the surrounding neighborhood individuals, of the mass media, of the government management, and of the social leaders. The propagation of a rumor or a truth is a process of a individual choice. It can continue to be convinced in the group. And the performance of the individual within the group take the action strategy to evolve in the panic behavior or exit the group(not following group's strategy).

\subsubsection{Interaction between individuals}

Referring to Coulomb's law, the degree of persistence of an individual's attitude toward collective panic behavior is analogous to the amount of charge in a vacuum neutral point charge, and the gap between individual attitudes is analogous to the distance between vacuum point charges, and the influence of individual attitudes is analogous to the interaction force between point charges, which reflects the change in attitudes towards panic behavior among individuals at the $t$ and $t+1$ moments. The influence function $S_{i}$ ' of individual $j$ on individual $i$ is set at the moment of $t$ :

$$
S_{i}^{\prime}=\sum_{j=1}^{N}\left(\frac{1}{N}\right) \frac{\left(1-P_{i}\right) P_{j}}{\sqrt{d_{i j}}}
$$

Among them, $P_{i}$ is the attitude persistence degree of individual $i, 1-P_{i}$ is the attitude shift degree of individual $i, \boldsymbol{P}_{i}$ is the attitude persistence degree of individual $i, d_{i j}$ is the attitude gap between individual $i$ and $j$, and $N$ is the number of neighbors that can be contacted by the peripheral area of individual $i$. When $d_{i j}=0$, the attitude gap between individual $i$ and individual $j$ is 0 , that is individual 
Wen ZHENG, Da ZHU; Simulation on Collective Panic Behavior Based on PI, Transactions on Networks and Communications, Volume 6 No. 6, December (2018); pp: 57-75

$i$ and $j$ have the same attitude towards collective panic behavior, while individual $i$ still adheres to its original attitude, that is $O_{i}(t+1)=O_{i}(t)$.

\subsubsection{Interaction between individuals and the mass media}

1) The extent to which the individual is influenced by the mass media is related to the authority of the mass media and the influence of the mass media. The influence function $S^{\prime}{ }_{M}$ of the mass media on the individual $i$ is:

$$
S^{\prime}{ }_{M}=\sum_{m=1}^{s} \frac{1}{S}\left(c_{1} Q_{m}(t)+C_{2} \operatorname{Im}(t)\right) \bullet\left(1-P_{i}(t)\right)
$$

Among them, $Q_{m}(t)$ is the authority of mass media, $I_{m}(t)$ is the mass media influence, and $S$ is the number of mass media involved in collective panic behavior. The influence of mass media on individuals is directly proportional to the authority of the mass media and the influence of the mass media. $C_{1}$ and $C_{2}$ are the ratio coefficients, $c_{1}+c_{2}=1$, which are set by weight.

2) The attitude of the mass media at the $t+1$ moment tends to be influenced by the management of the sudden panic behaviors at the $t$ moment, that is:

$$
O_{m}(t+1)=f_{1} O_{m}(t)+f_{2} M_{g}(t)
$$

Among them, $M_{g}(t)$ is the disposal efforts of the government towards to the sudden panic behavior of the group at the moment of $t, f_{1}$ and $f_{2}$ for the scale factor, $f_{1}+f_{2}=1$, and set by the weight. Because the mass media and individual belong to two different behavior subjects, the attitude gap between them is not taken into consideration.

\subsubsection{Interaction between individuals and social leaders}

The attitude of individual to collective panic behavior is influenced by the authority of leaders and the influence of leader, and the influence function $S^{\prime}{ }_{L}$ of the social leader on individual $i$ is :

$$
S^{\prime}{ }_{L}=\sum_{l=1}^{n} \frac{1}{n}\left(b_{1} Q_{l}(t)+b_{2} I_{l}(t)\right) \bullet\left(1-P_{i}(t)\right)
$$

Where $Q_{l}(t)$ is the authority of the social leader at time $t, \mathrm{n}$ is the number of social leaders in the group, $I_{l}(t)$ is the influence of the social leader at time $t$, Leadership authority and leadership influence are invariable, $b_{1}$ and $b_{2}$ are proportional factors, $b_{1}+b_{2}=1$, and are set by weight. Since social leaders and individuals belong to two different actors, the attitude gap between them is not taken into consideration.

\subsubsection{Interaction between individuals and government authorities}

1) The influence function $S_{G}{ }_{G}$ of government management on the individual attitude is:

$$
S^{\prime}{ }_{G}=\left(a_{1} M_{g}(t)+a_{2} C_{g}(t)\right) \bullet\left(1-P_{i}(t)\right)
$$


Transactions on Networks and Communications; Volume 6, No. 6, Dec 2018

Among them, $M_{g}(t)$ is the disposing of the government at time $t, C_{g}(t)$ is the government credibility in the time of $t, 1-P_{i}(t)$ stands for the transfer of individual attitude on the moment of $t, \boldsymbol{a}_{1}$ and $\boldsymbol{a}_{2}$ are the proportional factors, $a_{1}+a_{2}=1$. Because of the government departments and individuals belonging to two different subjects, therefore the gap between the two attitude will not be considered.

2) The credibility of the government at the next moment is influenced by the credibility of the government at the moment and the government's supervision of sudden panic behavior, that is:

$$
C_{g}(t+1)=e_{1} C_{g}(t)+e_{2} M_{g}(t)
$$

Among them, $\boldsymbol{e}_{1}$ and $\boldsymbol{e}_{2}$ are the ratio coefficient, $e_{1}+e_{2}=1$.

\subsubsection{Interaction between the group attitude and the group behavior}

The theorem of Kinetic Energy is used to describe the transition of individual behavior during the evolution of panic behavior. The individual attitude is analogous to the quality of the subject, the irrational degree of the individual is analogous to the velocity of the moving object, and the individual's behavior state is analogous to the kinetic energy of the object:

$$
B_{i}(t)=\left(1-O_{i}(t)\right) \cdot R_{i}{ }^{2}(t)
$$

Among them, $B_{i}(t)$ is the behavior state of the individual $i$ at the moment of $t, O_{i}(t)$ is the attitude of individual $i$ at the moment of $t$, and $R_{i}(t)$ is the irrational degree of the individual $i$ at the moment of $t$.

\section{MAS model in exploring PI's Impact}

Take the individual conformity as the research variable, and consider the individual characteristics and the external social environment characteristics in order to establish Multi-Agents simulation model. The agent has the ability to learn and can make independent decision of the individual, with closed twodimensional grid community in infinite space (which can carry a agent) to represent the spatial environment of agent, each agent can move in any direction and randomly, and they can adopt two kinds of behavior strategies: take part in panic behavior and do not participate in panic behavior. Among them, do not participate in panic behavior should include all the time not involved in the panic behavior, but also has been involved in panic behavior but now opt out of panic behavior. The criterion of formation of collective panic behavior is that people with relatively stable scale take part in the panic behavior.

There are four categories of subjects in the mimic system. These are: the individual, the mass media, the social leaders, the supervisor. In order to ensure the simulation effect of panic behavior, the Agents' attributes are the constant value, and the other values of the Agents' attributes are generated by the random number generator during the operation of the simulation.

Based on the simulation platform, the simulation model of collective panic behavior caused by rumors in emergencies is established. The model stimulate the influence of the initial population size(IPS), the initial population conformity(IPC), the proportion of the social leader's influence(PSL). $f$ is the promoted intensity, $g$ is the suppressed intensity, initial - participation is the initial participation rate of the panic behavior, and initial - conformity is the proportion of non-rational individuals in the population. 
Wen ZHENG, Da ZHU; Simulation on Collective Panic Behavior Based on PI, Transactions on Networks and Communications, Volume 6 No. 6, December (2018); pp: 57-75

\subsection{The Influence of promotion Intensity and suppression Intensity}

The effect of the promoted intensity $f$ and the suppressed intensity $g$ on the number of participants in the group panic behavior is shown in Fig. 2

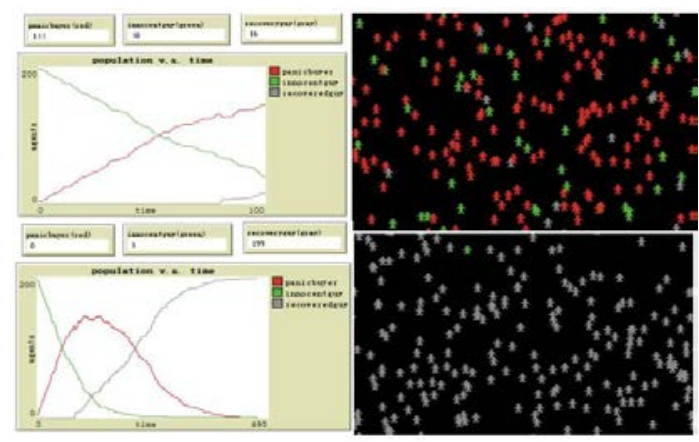

Fig. 2(a) Simulation results ( $f=0.5, g=0.25$ )

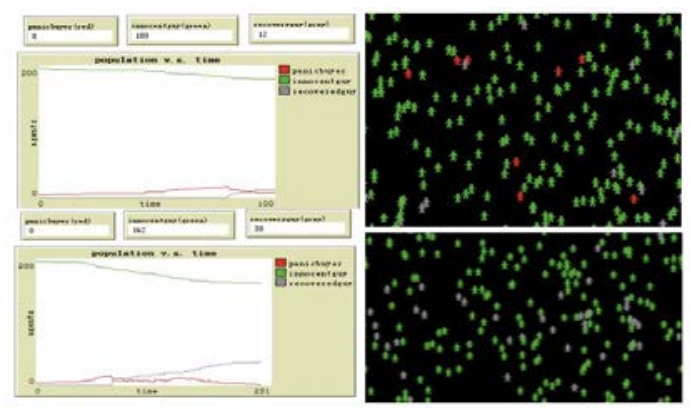

Fig. 2 (b) Simulation results ( $f=0.25, g=0.5$ )

when the behavioral strategies of the heterogeneous individuals in the population and the conformity of heterogeneous individuals are subject to the uniform distribution. When $f>g$, the number of the panic behavior in the crowd is greater than the number of the ones, who are not involved in the panic behavior, it indicates that the possibility of collective panic behavior is greater. When $f<g$, the number of panic behavior participant will reduce, interventions by government authorities have an inhibitory effect on the individual attitudes towards participating in the panic behavior. Part of the panic participants gradually withdraw.

Figure 2(a,b) effectively illustrates the control of the formation of the panic group. It is necessary in the initial stage of group in panic behavior to effectively control the spreading of the rumors. Those strategies are, to reduce the value of $f$, or to improve the degree of the government information disclosure, the government credibility, the government disposal efforts. In one word, that is, to increase the value of $g$. 


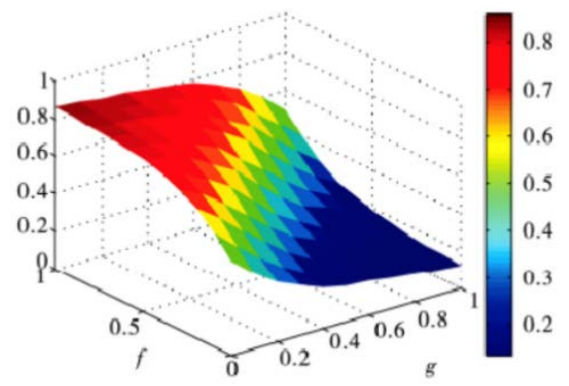

Fig. 3 The diagram of changing relationship in $f, g$, and participation rate

Figure 3 is a surface map, which indicates the participation rate of the group panic behavior changes with the promoting intensity and the inhibiting intensity. The abscissa represents the suppressing intensity of the behavior. The ordinate represents the promoting intensity of the behavior. The vertical coordinate represents the participation rate of the collective panic behavior in a stable stage. As it can be seen from the figure, even if the $f=0, g=1$, the participation rate of the group in the panic behavior is still greater than 0 . The reasons are those, not only of the behavior to promote the intensity, but also of the panic behavior to suppress the intensity. The panic behavior is also subject to the individual's own state features and the impact of such factors. In addition, with the promoting the intensity of the behavior, the participation rate of the group panic will remain at a certain level. Even the power of the behavior, which leads to suppress the intensity, is close to 1 . This is too tight in social structure because of the group in panic psychology leading to the whole. Although the intervention of the government management department controls intensity is too big, it cannot inhibit the formation of the collective panic behavior.

\subsection{The Initial Size of the Population(ISP)}

When $f=0.5, g=0.5$ is selected and the individual initial conformity characteristics obey the uniform distribution, the effects of initial panic behavior participation rate on the development trend of panic behavior are shown in Fig.4.

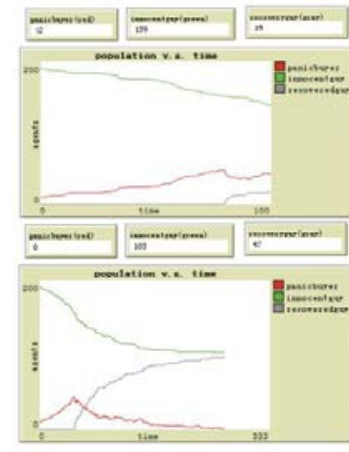

Fig. 4 (a) Simulation results $(f=0.5, g=0.5$, IPS rate $=\mathbf{5 \%}$

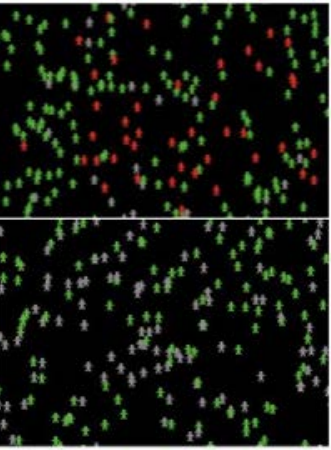

Fig. 4 (b) Simulation results ( $f=0.5, g=0.5$,IPS rate $=10 \%$ )

When the inhibitory strength of participants is changed for $g=0.25$ (Fig.6(a)) and $g=0.15$ (Fig.6(b)), the government intervention is not in time, resulting in the number of collective panic behavior of participants increased rapidly. Even much number of the agents don't participant panic, it have an initial share advantage. 


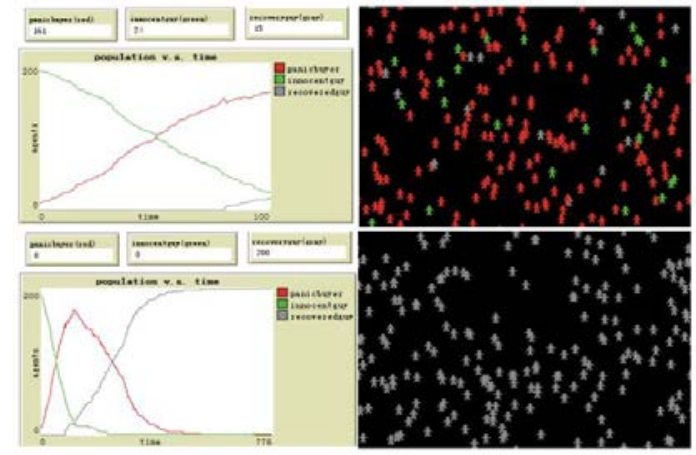

Fig. 5 (a) Simulation results $(f=0.5, g=0.25$, IPS rate $=5 \%$ )

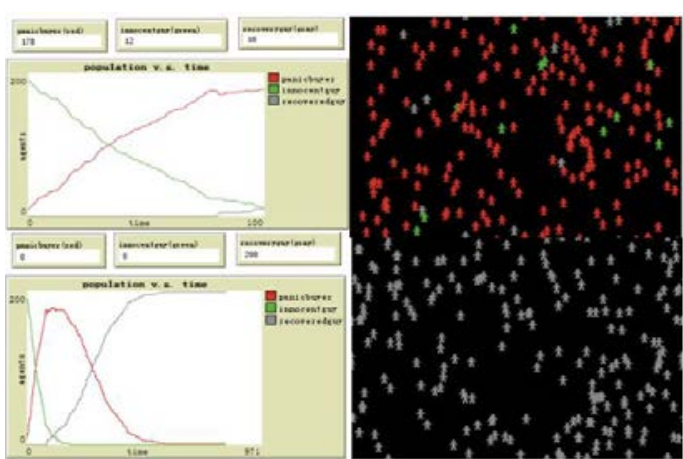

Fig. 5 (b) Simulation results ( $f=0.5, g=0.15$, IPS rate $=\mathbf{5 \%}$ )

It shows that the expanding of panic behavior is affected by the participation rate of the initial participants in space in fig.6, and the impact of government intervention on panic behavior. Even if the scale of the initial participation of collective panic behavior is not very large, it will lead to the scale of panic behavior expanding rapidly. And more people will involve in, if the government does not intervene.
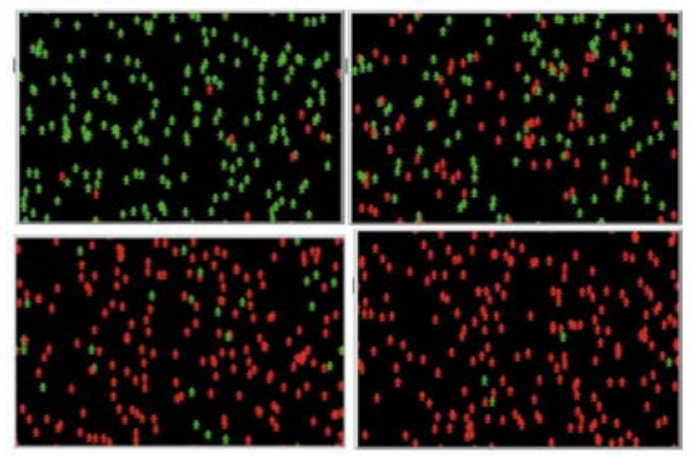

Fig. 6 simulation results (IPS rate $=\mathbf{5} \%$ to $98 \%, f=0.5, g=0.25$ )

\subsection{The Initial Population Conformity(IPC)}

Simulation parameters $f=0.5, g=0.25$, when the individual's initial behavior strategy is evenly distributed, the influence of individual conformity characteristics on the evolution results is revealed.

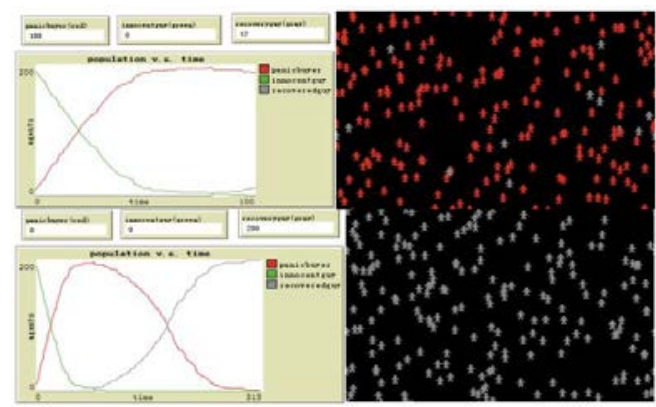

Fig. 7 (a) Simulation results $(f=0.5, g=0.25$, IPC $=\mathbf{2 5 \%}$

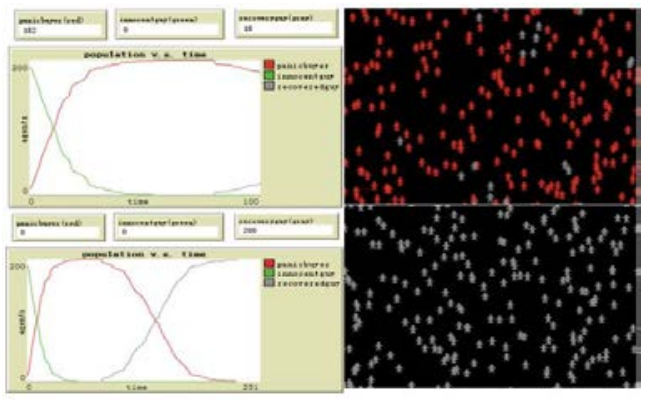

Fig. 7(b) Simulation results

( $f=0.5, g=0.25$, IPC=50\%) 
Transactions on Networks and Communications; Volume 6, No. 6, Dec 2018

It can be seen from the figure that under the same conditions, the proportion of irrational individuals in the initial population is greater, when the panic behavior involves a strong herd. In addition, Figure 7 (b) and Figure 2 (a) are two results of the simulation parameters of the same, the initial population size under the different conditions. By contrast, initial population conformity expands the scale of the participates in panic behavior. This is because when there are more non-rational individuals in the space group, the individual behavior strategy choice is more inclined to dominate the behavior strategy of most people. In this simulation experiment, due to the strength of promoting the panic behavior less than the strength of inhibiting one. The collective panic behavior after the short-term evolution was more dominant, so the individuals, who has herd characteristics, are more likely to take part in the panic.

\subsection{The Proportion of Social Leaders(PSL)}

Simulation parameters $f=0.5, g=0.25$, and when the individual's initial behavior strategy is distributed evenly, the influence of the proportion of social leaders in the group on the evolution results is considered. As the following Fig. 8 show.

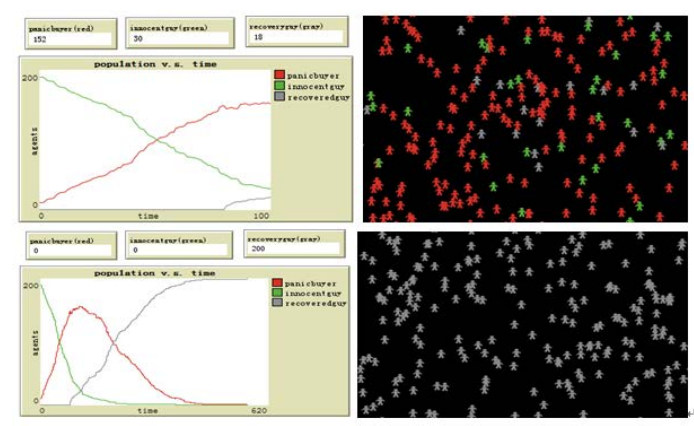

Fig.8(a) Simulation results ( $f=0.5, g=0.25$, PSL= 5\%)

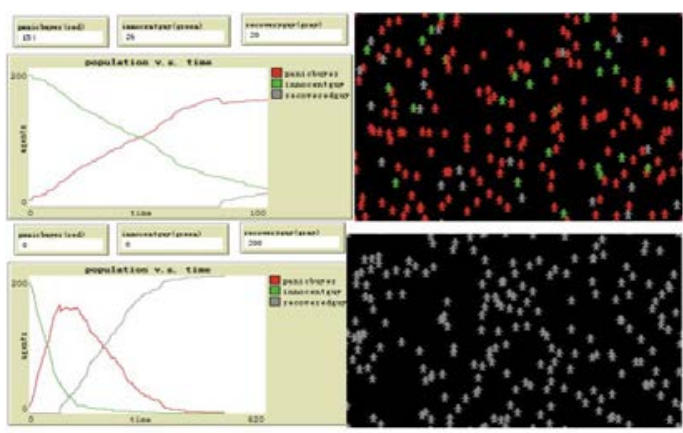

Fig. 8(b) Simulation results ( $f=0.5, g=0.25$, PSL=10\%)

It can be seen from the figure that under the same conditions, the proportion of social leader in the crowed is greater. When the leader was infected and transformed into the participants of panic behavior, due to its great social influence, more people around to spread rumors and related rumors. It results that more not involved are infected and turned into the panic participants. The expansion of the group panic is affected rapidly. And when social leaders are exposed to the truth and withdraw from the group panic behavior, because of its social influence, there will be more people around them will clarify the facts. The numbers of the collective panic behavior participants fall sharply and the panic behavior will gradually ease.

\section{Discussions and Conclusions}

The simulation results show the context of an accident. When the panic behavior occurred, which is caused by the rumors, the more the initial participation of collective panic behavior, the more conducive to induce the group spreading the rumors. The characteristics of the individual conformity can cause individuals, who have not yet participated in the panic behavior to support the dominant position of the party between promoting and suppressing the intensity of the panic behavior. If the proportion of social leaders in the crowed is great, the leader is infected and transformed into the participants of the panic behavior. Due to his(her) large social influence and wide network of connection, the more people around him are likely to spread the rumors, the more people are infected and transformed into the panic 
Wen ZHENG, Da ZHU; Simulation on Collective Panic Behavior Based on PI, Transactions on Networks and Communications, Volume 6 No. 6, December (2018); pp: 57-75

participants. It leads to the scale of behavior in expanding the panic rapidly. Then the number of the panic behavior participants increased sharply. Furthermore, when the social leaders are exposed to the truth of the incidents and withdraw from the collective panic behavior, because of its social influence and wide social net connection, they will clarify the facts to more people. The rumors are self-defeated. The number of collective panic behavior participants declines sharply. Therefore, the collective panic behavior is gradually eased and subsided.

$f(r, e)$ and $g(c, l)$ in the evolution model represent two different "powers", which are the promotion and the suppression of the collective panic behavior. The regulation mechanism of the collective panic behavior caused by rumor can be considered in the following two aspects:

1. In the early stages of panic behavior, it should effectively reduce the intensity of rumor dissemination to achieve the purpose of $f(r, e)$.

2. Because of the low degree of controllability of rumor transmission, it needs a lot of auxiliary management tools and measures. Therefore, in the actual macro- management, scheme 1 is often difficult to implement in practice. In order to truly advance the prevention and effective control of the collective panic behavior, it also can improve the government's credibility and coping with crisis disposal efforts.

\section{ACKNOWLEDGMENTS}

The authors gratefully acknowledge the financial support from the NSFC (National Natural Science Foundation of China,71473032) and CSC(China Scholarship Council,201508130039) .

\section{REFERENCE}

[1] Barabási AL, Albert R. Emergence of scaling in random networks. Science 1999;286:509-12.

[2] Bergemann D, Valimaki J. Learning and strategic pricing. Econometrica 1996;64(5):1125-49.

[3] Bi Hongyin (2007).Analysis of the Group Influence in the Formation and Change of Network Public Opinion[J].Journal of TianJin University: (Social Sciences Edition),9(3):270-273.(in Chinese)

[4] Carlos Lemos, Rui Jorge Lopes, Helder Coelho (2016). On Legitimacy Feedback Mechanisms in Agent-Based Modeling of Civil Violence[J]. International Journal of Intelligent Systems, Vol.31,106-127.

[5] Chang Qin, Dang Huisen (2011). Research on population clustering model based on grid agent[J]. Journal of the People's Public Security University(Natural Science Edition), 17(3):71-74. (in Chinese)

[6] Chen Peng, Chen Jianguo (2015).Research on Multi - Agent Modeling of Sudden Group Violence[J].Computer Simulation, 32(1):377-381.(in Chinese)

[7] Chen Shiming, Huang Jiangping, Jiang Hong (2007). Research on modeling and simulation of swarm behavior in uncertain environment[J]. Computer Engineering and Applications, 43(21):32-35. (in Chinese) 
Transactions on Networks and Communications; Volume 6, No. 6, Dec 2018

[8] Dai Wei, Yu Lean, Tang Ling, Shen Yan (2015).A Study on the Public Information Strategy of Public Panic in Unconventional Emergencies: Based on Multi-Agent[J]. System Engineering-Theory \&Practice, 35(3):641-650.(in Chinese)

[9] Du Rong, Liang Hongxia (2011).Simulation Government's Guide Effect on Network Opinion of Public Crisis[J]. Journal of Intelligence, 30(11):61-66. (in Chinese)

[10] Du Rong, Liang Lei,Wang Ning (2015). A Simulation Study on the Mass Emergency Evolution Based on the Social Physics--Evolution of Community Advice and Behavior[J].Complex Systems and Complexity Science, 12(3):61-69.(in Chinese)

[11] Edward Chow, Jonathan Elkind (2005). Hurricane Katrina and US Energy Security[J].Survival, 47(4):145-160.

[12] He Xiaoyuan, Hu Xiaofeng, Si Guangya (2010). Research on network information communication behavior based on social physics[J]. Journal of System Simulation, (12):2957-2962.(in Chinese)

[13] Izquierdo SS, Izquierdo LR, Galán JM, Hernández C(2005). Market failure caused by quality uncertainty. In: Mathieu P, Beaufils B, Brandouy O, editors. Artificial economics - lecture notes in economics and mathematical systems 564. Berlin: Springer-Verlag; 2005.

[14] Kirman AP, Vriend NJ (2001). Evolving market structure: an ACE model of price dispersion and loyalty. Journal of Economics Dynamic Control. 25(3-4):459-502.

[15] Kou Gang, Zhao Yiyi, Peng Yi (2012).Modeling and Analysis of Snapping Behavior under Sudden Incident[J]. Chinese Journal of Operations Research,20(1):85-91.(in Chinese)

[16] Li Huaqiang, Fan Chunmei, Jia Jianmin (2009). Public Risk Awareness and Emergency Management in Sudden Disasters: A Case Study of 5.12 Wenchuan Earthquake[J]. Management World, (6):52-63.(in Chinese)

[17] Li Lanying (2008).Based on CA's network of public opinion factors[J]. Science Technology and Engineering, 8(22):61796183.(in Chinese)

[18] Li Naiwen, Liu Yi, Huang Min (2014).A Study on Influencing Factors of Intrinsic Deviation in Individual Risk in Unconventional Emergencies[J]. Journal of China Safety Science, 24(5):10-14.(in Chinese)

[19] Liu Jia, Xie Kefan (2013).Study on influential factors of individual decision-making behavior of unconventional emergencies[J]. Soft Science, 27(3):50-54.(in Chinese)

[20] Liu Junguang, Ou Wei (2014). Research on Emergence Model and Simulation of Rumor Propagation of Sudden Incident[J]. Military Operations Research and Systems Engineering, 28(3):66-69. (in Chinese)

[21] LuQiaoyu, Shao Bo (2011).Analyzing public emergencies in the main behavior model based on the system thinking[J]. Public Management,(10):149-152.(in Chinese)

[22] Qiang Chaohua, Wu Peng (2014).A Study on the Behavior of Internet Users in the Process of Public Opinion Evolution of Sudden Events[J].Journal of Information Analysis and Research,(6):71-78.(in Chinese)

[23] Sun Duoyong (2006). The Economic Analysis on the phenomenon of individual buying in emergencies[J]. Economy and Management, (11):208-231.(in Chinese)

[24] Thomas A.Glass, Monica Schoch-Spana (2002). Bioterrorism and the People: How to Vaccinate City against Panic[J].Clinical Infectious Disease,34(2):217-223. 
Wen ZHENG, Da ZHU; Simulation on Collective Panic Behavior Based on PI, Transactions on Networks and Communications, Volume 6 No. 6, December (2018); pp: 57-75

[25] Virginia Giorno, Serena Spina (2016). Rumor Spreading Models With Random Denials[J]. Physica A, Vol.461,569-576.

[26] Wei Jiuchang, Zhou Lei, Zhou Xin (2011). A Study on the Evolving Mechanism of Group Snapping in Public Crisis - A Case Study of Salt Selling in China Based on Japan's Nuclear Crisis[J]. Journal of management Case Study, 12(6):478486.(in Chinese)

[27] Xie Liren, Chen Junmei, Zhang Minqin (2013).Research on influencing factors of sudden snapping behavior[J].Journal of XiAn University of Technology, 33(7):572-576.(in Chinese)

[28] Xu Zhiyu (2003).Compensation structure leads to control transfer - an analysis of SARS induced buying behavior [J]. Journal of FuZhou University (Philosophy and Social Sciences), (4):9-11.(in Chinese)

[29] Yang Zhimo, Si Guangya, Li Zhiqiang (2010).Design and implementation of large - scale group behavior simulation model[J].Journal of System Simulation,22(3):724-727.(in Chinese)

[30] Yuan Guiqiu, Pu Guohua (2005). Theoretical Explanation in Panic Buying[J]. Statistics and Decision, (1):56-57. (in Chinese)

[31] Zhang Fang, Si Guangya, Luo Pi (2011).A rumored propagation simulation model based on communication function[J].Journal of System Simulation, 23(11):2482-2486.(in Chinese)

[32] Zhang Yaofeng, Xiao Renbin (2014).Emergence mechanism of consensus synchronization in internet collective behavior based on cellular automata[J]. System Engineering - Theory \& Practice, 34(10):2600-2608. (in Chinese)

[33] Zhang Yaofeng, Xiao Renbin (2015).Model and simulation of consensus evolution mass emergency[J]. Application Research of computers,32(2):351-355. (in Chinese)

[34] Zhao Laijun, Cheng Jingjing (2010). Research on Individual Behavior State under Unconventional Sudden Incident Based on Mutation Theory[J]. Journal of China Safety Science, 20(12):14-19. (in Chinese)

[35] Zhao Yiyi, Peng Yi, Xiao Lei (2015). Study on the Mechanism of Public Opinion Propagation of Group Snapping in Sudden Incident[J].System Engineering-Theory \& Practice, 35(3):616-622. (in Chinese)

[36] Zheng Xiaojing, Zheng Junjun (2016).Criticality of symmetry breaking of collective behavior driven by Individual rules[J].System Engineering-Theory \& Practice, 36(2):413-426. (in Chinese)

[37] Zhuang Yaming, Yu Hailin (2013). Research on Characteristics of Information Propagation Network of the Mass Unexpected Incident: A Case Analysis of Panic Buying of Candles[J]. Journal of Intelligence,32(7):37-42.(in Chinese) 
turtles-own [

infected? ;;if true, the person is either a panic buyer or a recovered guy.

panicbuyer? ;; if true, the person is participating in the panic buying event.

socialleader? ;; if true ,the person is a social leader which means he has greater influence on others.

probability-to-infect ;; the probability of a normal guy to get infected

probability-to-recover ;; the probability of a abnormal guy to get recovered

]

ii;

;i; SETUP PROCEDURES

ii;

to setup

clear-all

askpatches [ setpcolorblack ]

set-default-shapeturtles"person"

setup-people

;; the original source rumor infectors of the whole panic buying event

askn-of ( round ( initial-participation /100* initial-people ) ) turtles [

set infected? true

set panicbuyer? true

assign-color ]

askn-of ( round ( proportion-of-social-leader /100* initial-people ) ) turtles [

set socialleader? true ]

reset-ticks

end

to setup-people

create-turtles initial-people

[ setxyrandom-xcorrandom-ycor

set infected? false

set panicbuyer? false

set socialleader? false

setsize1.5;; easier to see

assign-color

set probability-to-infect 0

set probability-to-recover 0

]

end

to assign-color ;; turtle procedure

ifelsenot infected?

[ setcolorgreen ] ;; it indicates that the person has not yet participated in panic buying event.

[ ifelse panicbuyer?

[ setcolorred ] ;; it indicates that the person is participating in panic buying event.

[ setcolorgray ] ] ; it indicates that the person has recovered from the panic buying event.

end

;i;

;i; GO PROCEDURES

ii;

to go

askturtles [ move ]

getinvolved

government-interfere

getrecovered

askturtles [ assign-color ]

Copyright (c) Society for Science and Education, United Kingdom 
Wen ZHENG, Da ZHU; Simulation on Collective Panic Behavior Based on PI, Transactions on Networks and Communications, Volume 6 No. 6, December (2018); pp: 57-75

tick

;; if ticks = 100 [ stop ] ;; to get the temporary view and plot on the fly when ticks equal to one hundred.

;; when the count of panicbuyer less than or equal to the $1 \%$ of initial-people,

;; we can almost think the panic buying event is over.

if ( ticks >= government-interfere-time ) and ( notany?turtleswith [ panicbuyer? =true ] )

[ stop ]

end

;; people move about at random

to move ;; turlte procedure

rtrandom-float360

Itrandom-float360

fd1

end

to getinvolved

;; because of touching rumor infectors, innocent guy have the probability1 to become a panic buyer

askturtleswith [ infected? =false ]

[

ifrandom-float $2<$ probability1

[ set infected? true

set panicbuyer? true ]

]

end

to government-interfere

;; to research how to peace the panic buying behaviour, we start to consider this factor here

if ( ispublicized ) and ( ticks= government-interfere-time )

[ askn-of ( round ( government-credibility /100* initial-people ) ) turtleswith [ panicbuyer? =true ]

[ set panicbuyer? false ]

]

end

to getrecovered

;; because of touching truth, panic buyer starts to get recovered

ifany?turtleswith [ infected? =trueand panicbuyer? =false ] [

askturtleswith [ panicbuyer? =true ]

[

ifrandom-float $2<$ probability 2

[ set infected? true

set panicbuyer? false ]

]

]

end

;; the innocent guy has the probability1 to become a panic buyer

to-report probability1

askturtleswith [ infected? =false ] [

let number-a 0

let number-b 0

ifelse socialleader? =false

[ set number-a ( count ( turtles-onneighbors4 ) with [ panicbuyer? =true ] )

set number-b ( countturtles-onneighbors4)

URL: http://dx.doi.org/10.14738/tnc.66.5740 


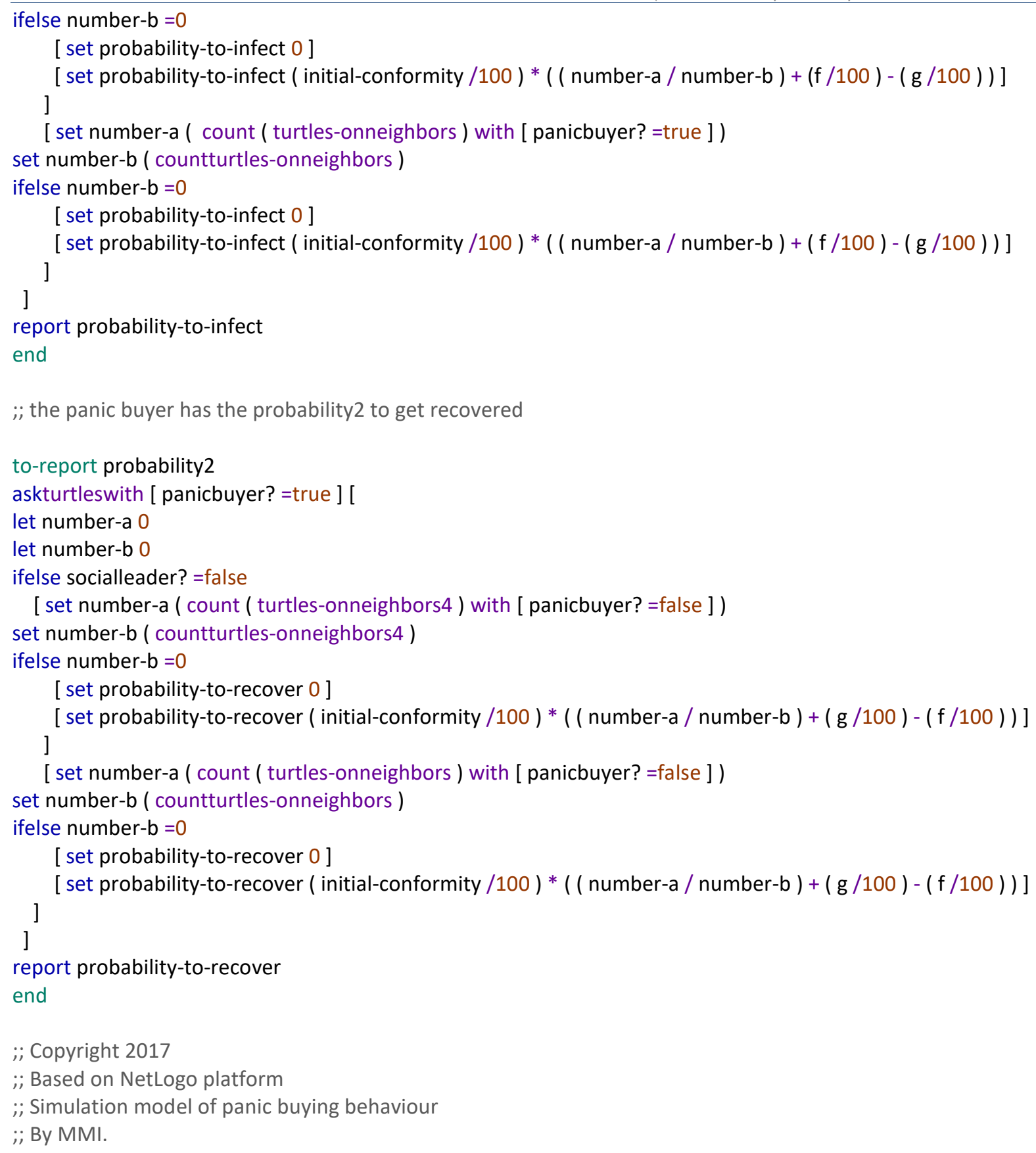

